\title{
Educación sensible: efectos de una pedagogía formativa para la convivencia democrática en escolares peruanos
}

Sensitive education: effects of a formative pedagogy for democratic coexistence in Peruvian schoolchildren

Volumen 19, Número 3

Setiembre-Diciembre

pp. 1-24

Lucero Estrella Alcántara Mego Jhon Alexander Holguin Alvarez

\section{Citar este documento según modelo APA}

Alcántara Mego, Lucero Estrella y Holguin Alvarez, Jhon Alexander. (2019). Educación sensible: efectos de una pedagogía formativa para la convivencia democrática en escolares peruanos. Revista Actualidades Investigativas en Educación, 19(3), 1-24. Doi. 10.15517/aie.v19i3.38628 


\title{
Educación sensible: efectos de una pedagogía formativa para la convivencia democrática en escolares peruanos
}

Sensitive education: effects of a formative pedagogy for democratic coexistence in Peruvian schoolchildren

\author{
Lucero Estrella Alcántara Mego ${ }^{1}$ \\ Jhon Alexander Holguin Alvarez ${ }^{2}$
}

\begin{abstract}
Resumen: El artículo se elaboró como respuesta a la falta de convivencia democrática social que se ha trasladado al sistema educativo peruano. Los hallazgos, desde el año 2015 hasta la actualidad, indican que este problema prevalece en los grupos escolares organizados por políticas con falta de justicia social. Adicionalmente, el uso de una pedagogía vertical produce un estado anárquico en el aula. Este estado anárquico primigenio se transfiere entre docentes y estudiantes mediante la ejecución de la programación curricular, razón por la cual el problema parte del mismo proceso de escolarización pública. Como objetivo central se planteó comprobar el cambio democrático escolar de la convivencia luego de aplicar un programa de Educación Sensible o Educación desde la sensibilidad, el cual es una teoría pedagógica apoyada en la formación moral para fortalecer acciones basadas en la cooperación social. La metodología de la investigación tiene un enfoque cuantitativo, con tipología de diseño preexperimental. La muestra estuvo conformada por 40 estudiantes de cuarto grado de primaria ( $M=$ 10.1 años; D.E. = 1.84), originarios de un contexto vulnerable de Lima, Perú. Se utilizó la Escala de Medición de la Convivencia Democrática (ESMCD). Los resultados corroboran que el método de Educación Sensible basado en concientización, autonomía y autoevaluación emocional incrementaron los índices de convivencia democrática. Se concluye que los factores de autoconciencia, reflexividad, y sensibilidad actitudinal y afectiva orientan y determinan la constitución de los valores democráticos materializados en indicadores como (a) capacidad de tolerancia, (b) ajuste a las normas democráticas y (c) resolución de conflictos. Por último, la reflexión sobre los resultados permite indicar que la colaboración es la competencia que se desarrolló con mayor lentitud en el grupo estudiantil.
\end{abstract}

Palabras clave: autoevaluación, tolerancia, educación sensible, convivencia democrática.

\begin{abstract}
The article was developed in response to the lack of social democratic coexistence, which has been transferred to the Peruvian educational system. The findings from 2015 to the present point that this problem prevails in school groups organized by policies with a lack of social justice. Additionally, the use of a vertical pedagogy produces an anarchic state in the classroom. This primal anarchic state is transferred between teachers and students through the execution of curricular programming, so the problem starts from the process of public schooling. The central objective was to verify the democratic school change of coexistence after applying a program of Sensitive Education or education based on sensitivity, which consists of a pedagogical theory supported by moral training to strengthen actions based on social cooperation. The methodology of the research has a quantitative approach, with a pre-experimental design typology. The sample consisted of 40 individuals from the fourth grade of primary school $(M=10.1$ years, D.E. = 1.84), from a vulnerable context in Lima, Peru. The Scale of Measurement of Democratic Coexistence (ESMCD) was used. The results corroborate that the method of Sensitive Education based on awareness, autonomy and emotional self-evaluation increased the rates of democratic coexistence. It is concluded that the factors of self-consciousness, reflexivity, and attitudinal and affective sensitivity guide and determine the constitution of democratic values materialized in indicators such as: a) tolerance capacity, b) adjustment to democratic norms and c) conflict resolution. Finally, the reflection on the results, indicate that collaboration is the competition that was developed more slowly in the student group.
\end{abstract}

Keywords: self appraisal, tolerance, sensitive education, democratic coexistence.

\footnotetext{
1 Docente de la Universidad César Vallejo, Facultad de Educación e Idiomas. Lima, Perú. Dirección electrónica: luceroalcantara16@gmail.com ORCID https://orcid.org/0000-00017157-4325
}

2 Docente de la Universidad César Vallejo, Facultad de Educación e Idiomas. Lima, Perú. Dirección electrónica: jhonholguinalvarez@gmail.com ORCID https://orcid.org/0000-0001$\underline{5786-0763}$

Artículo recibido: 19 de marzo, 2019

Enviado a corrección: 22 de mayo, 2019

Aprobado: 10 de junio, 2019

Los contenidos de este artículo están bajo una licencia Creative Commons 


\section{Introducción}

La perspectiva social sobre democracia se ha confundido en la actualidad. Las diferentes concepciones políticas, sociales y culturales han diversificado tanto su concepción entre la población latinoamericana que se han forjado líneas de pensamiento fuera de los límites del pensamiento ético humano. Los efectos de maduración de esta perspectiva de democracia sobre sus habitantes influyen en la estructura social de las naciones suramericanas. Las diferentes formas de pensar se transfieren a algunos aspectos humanos sociales importantes: derecho, actitudes, pensamiento. Desde estas diferentes perspectivas en la convivencia, los conceptos de vivir y convivir se han transformado debido a las influencias sociodemográficas y políticas constructivas del concepto democracia. Este hecho es patente, observable y se evidencia en problemas particulares como corrupción, traición, delitos políticos, denigración incluso manifestaciones de violencia como matanzas o extorsiones. El reflejo de estos problemas sociales se manifiesta mediante el currículo en la educación. Inclusive, el mismo concepto de educación no está exento del debate de la convivencia, en especial, la convivencia escolar democrática en el aula, ya que en esta se transmiten formas de convivir preconfiguradas en los escolares durante la adopción de comportamientos en el consumo de diferentes fuentes de información (televisiva, radial o redes de internet).

La convivencia democrática es una competencia social. En principio, los niños y las niñas respetan el cambio, la adopción de ideas, el cumplimiento de deberes y las interrelaciones, los cuales coinciden en el desarrollo ético, socioafectivo e intelectual. Este constructo es complejo en cuanto a su estructuración cognitiva en el aula; y más aún, su práctica y adopción formativa. Respecto de este punto, Colcord, Mathur y Zucker (2016) evidenciaron que el constructo de convivencia democrática a nivel cognitivo difiere de su efectividad como una iniciativa en el desarrollo del alumnado mediante interacciones en el aula. Un año más tarde, Redford, Desrochers y Hoyer (2017) estableció que la democracia requiere de mejoras en su inclusión política, la cual resuelve problemas en la formación de ciudadanía, género, tolerancia e interculturalidad. De esto se coligió que antes de abordar la asimilación y prácticas democráticas en el aula, se debe repensar el sentido político de la democracia en las escuelas. Así, se puede asumir que las políticas educativas, el currículo y la programación curricular necesitan de un engranaje transversal para democratizar las actitudes estudiantiles en su convivencia. 
Un estudio internacional de la Asociación Internacional para la Evaluación de Logros Educativos (ICCS, 2016), que evaluó parte de la convivencia en democracia en Educación Cívica y Ciudadanía, identificó al $21,3 \%$ de estudiantes peruanos como efectivos participantes en actividades cívico-democráticas en sus instituciones educativas. Por su parte, el Proyecto Educativo Metropolitano (PEM, 2014) indicó que solo el 31,2 \% de escolares promovía y participaba en la conformación de Municipios Escolares. Ante esta realidad, en los distritos de Lima, se han establecido formatos de atención de la convivencia democrática desde un enfoque aplicado en el aula como respuesta a los resultados de la ICCS. Sin embargo, el establecimiento de estas propuestas desde el Ministerio de Educación del Perú (MINEDU), demuestra ciertas deficiencias: en algunos casos, las estrategias de cada docente son muy verticales y aún tratan el ingreso informacional del significado de democracia sin practicarla. El sentido común de elegir se aprende desde la realización de pertenencia al grupo, el respeto por las decisiones, la elección adecuada y la formulación de ideas para mejorar otros campos. En dicha práctica es necesario el conocimiento del otro, tanto entre estudiantes como en la relación profesorado-alumnado.

La prioridad en educación debe ser resolver el desconocimiento de cada estudiante en sus distintos aspectos. Esta es una de las falencias que este tipo de propuestas masivas aplicadas en las instituciones educativas descuidan o no internalizan en sus procesos de gestión. Las prioridades educativas en la actualidad se centran en el acompañamiento del estudiantado desde acciones como conocimiento de la preocupación estudiantil, percepción de su mundo interactivo, su interacción física y su concepción sobre el ejercicio de la moral, las cuales se consideran bases para una Educación Sensible (ES). Esta perspectiva buscó el desarrollo de los ejes formativos en el estudiantado mediante la formación social en contextos democráticos saludables.

\section{Referentes teóricos}

\subsection{Educación sensible desde la propuesta de Herbart}

La sensibilidad permite comprender la otredad, los sentimientos y los pensamientos, e interpretar la conducta de los sujetos del entorno como componentes de la sociedad (Herbart, en Touriñan, 2013). La educación sensible es una doctrina pedagógica de instrucción de la moral para fortalecer las acciones virtuosas y sensibles en cooperación social. Desde este enfoque, se concibe al ser humano como el sujeto que realiza mejores interacciones con otros si ha aprendido a desarrollar habilidades para desarrollar el 
conocimiento. En dicha interacción interviene la proyección de un comportamiento determinado por la cultura, por la construcción social del ser (Vygotsky, en Schunk, 2012). Por esto, la educación sensible no es individualista, es pragmática y humanista en tanto desarrolla la estructura social democrática en la interacción social del estudiantado. Este desarrollo sociocultural en las áreas humanística y pragmática permite el incremento de la sensibilidad como andamiaje de otros enfoques de convivencia, como el de naturaleza directa, ministerial, reactivo o proactivo.

La educación sensible se basa en el proceso de sensibilidad en las interrelaciones estudiante-estudiante y docente-estudiante en la escuela, y se sustenta en el desarrollo del tacto sensible para entender ideas, conceptos, actitudes; saber percibir acciones y actuar en la vida cotidiana escolar. El acto de percibir estímulos internos y externos es clave para el colectivo docente en las relaciones con estudiantes (Lowe, 2015), tanto que dichos estímulos se enlazan con mayor empatía a los componentes socioafectivos, conductuales y del pensamiento para la formación de sociedades escolares más justas, más perceptibles; en suma, reflexivas. La educación sensible es la capacidad de interacción física y moral entre estudiantes con afinidad humana (Bickmore, Salehin Kaderi y Guerra-Sua, 2017; Lowe, 2015). De ella se desarrollan los componentes que permiten comprender, asumir diversos roles por parte de un grupo y resolver conflictos de forma democrática. Comprende acciones centrales en la búsqueda de la conciencia, adquisición progresiva de la autonomía, autoevaluación de las emociones y de las acciones como producto moral. Estos tipos de mecanismos desde la formación permiten modificar la valoración del estímulo sensible y responder a situaciones democráticas determinadas.

Al respecto, algunos estudios encontraron efectos en la coexistencia pacífica luego de desarrollar intervenciones pedagógicas basadas en la comprensión de problemas sobre participación democrática (Gass Gough, Armas, y Dolcino, 2016; Jakobus Jacobs, 2015). Otros hallazgos evidencian que la resiliencia influye directamente en el comportamiento violento, el cual reduce en el aula (Colcord et al., 2016; Gass et al., 2016). En otros casos, se piensa que algunos tipos de comportamiento negativo (agresividad, conflicto, disrupción) suelen impedir el desarrollo de la sensibilidad cuando, por lo contrario, la práctica de dicha situación permite formar a estudiantes con conductas conflictivas (Lowe, 2015). Esto se debe a que la expresión vivencial y reflexiva del comportamiento negativo fortalece el carácter endeble y lo convierte en una fortaleza humana que facilita la comunicación de convivencia. 
Finalmente, otros indicios empujan a formular que el conocimiento democrático en la población estudiantil de contextos vulnerables es flexible, siempre y cuando el enfoque de educación sensible también sea flexible, si se verifican los poderes de negociación más próximos del entorno estudiantil tanto en tareas científicas o académicas (Chang y Song, 2016). El poder de negociación en la educación sensible también influye en la transformación social (Aslan, 2018; Bickmore et al., 2017; DeAngelis, 2017). Algunas características de educación sensible están asociadas a la tolerancia, la negociación de conflictos, la toma de decisiones proactivas y al abordaje de interrelaciones imprácticas.

\subsection{Enfoque democrático de la convivencia en el aula}

La escuela democrática concebida por Dewey (1916) caracterizó el comportamiento democrático como el conjunto de competencias cívicas de los seres humanos perteneciente a una estructura social. Este conjunto de competencias cívicas implica tantos comportamientos equitativos de democracia como comportamientos de inequidad. Consideró que el ser humano logra su libertad si diferencia en la práctica social su propio comportamiento, aunque este sea negativo, con el fin de establecer un orden social. Extrapolando, la convivencia democrática escolar implica la participación de todos bajo decisiones normativas establecidas en consenso. Todos se desarrollan influyendo con sus factores interpersonales, culturales y sociales (in situ) en sociedad bajo prácticas valorativas o axiológicas.

La convivencia democrática es la interrelación entre los participantes e integrantes activos que, como miembros sociales del aula, se adhieren a un proceso educativo determinado bajo las normativas postuladas y aceptadas en consenso (Rendón et al., 2016). Desde esta perspectiva, los derechos y normas influyen en la constitución de las relaciones interpersonales (Muñoz Quezada, Lucero Moncada, Cornejo Araya, Muñoz Molina y Araya Sarabia, 2014), con el fin de hacerlas más compactas y duraderas, en base a las necesidades individuales y sociales que surgen para la construcción de la paz (Aguilar, Peter de Bran y Aragón, 2014). Por lo tanto, una convivencia democrática se concibe como el establecimiento de relaciones prácticas entre pares, prácticas sociales en las escuelas, asunción de roles, tareas y cooperación para la cohabitación democrática. 


\subsection{Hallazgos e hipótesis desde la teoría democrática}

La convivencia democrática en las escuelas, especialmente en aquellas que están ubicadas en contextos de conflicto social, ambientes de peligro y exclusión colectiva, ya es un desafío para el colectivo docente, en primer lugar, para aprender a convivir democráticamente desde el planteamiento de políticas para la coexistencia en el aula y ejercer las funciones normativas en ese espacio (Bowie, 2017; Grau, García-Raga y LópezMartín, 2016; Grau y García-Raga, 2017). Respecto a la intervención democrática, cada escolar con escasa participación en la escuela, por lo general, realiza actividades que carecen de aspectos motivacionales, lo cual desfavorece la integración total del grupo. Este hecho ha sido profundizado en distintas investigaciones (Alaníz Castro, Morán López y Blandón Blandón, 2016; Berlowitz; Frye y Jette, 2017; Eriksen, 2018; Gass et al., 2016) que señalan que la ciudadanía social depende de las relaciones integradoras. Desde el enfoque de formación democrática de la convivencia, los hallazgos postulan que la autonomía emocional es un acrecentador del comportamiento democrático debido al uso proactivo de normas (DeAngelis, 2017) con el fin de crear conciencia sensible. El proceso cultural y de cohesión entre los participantes de un aula es más fuerte si la pedagogía formativa se adecúa a las formas de convivencia escolar (Grau y García-Raga, 2017). De igual forma, el ser autónomo y consciente en el aula debe ser capaz de razonar sobre sus propias acciones, independizar su autoevaluación emocional y sensibilidad ante los demás (Samuelsson, 2016).

DeAngelis (2017) planteó que la coexistencia promueve la formación holística. Sin embargo, en algunos contextos, las normas culturales y sociales originadas en dicha coexistencia influyen en la construcción de entornos escolares saludables. Estos, por estructurarse holísticamente, necesitan retroalimentarse en la práctica vivencial con el propósito de hacerlas para escuelas democráticamente sostenibles. El objetivo de la convivencia para una educación democrática sostenible debe superar la aparición de otras variables concurrentes, como la deserción o, en su defecto, posiciones anárquicas emergentes en el alumnado, es decir, la propia pedagogía para la convivencia necesita formar al estudiantado para su egreso de la escuela y su futura inclusión en la sociedad actual.

Por todo lo vertido hasta aquí, el estudio buscó comprobar si el cambio democrático de la convivencia democrática escolar puede ser más flexible en sus componentes si se analizan los aspectos sensibles del ser humano escolar mediante: (a) concientización 
sensible, (b) búsqueda de autonomía, (c) realización de autoevaluación emocional y (d) la sensibilidad aplicada a la convivencia. Por ello, se promovió la medición de los efectos de estas acciones mediante una pedagogía formativa de Educación Sensible (ES) en un programa de intervención de la convivencia democrática en un grupo de escolares que asistía a una escuela ubicada en un contexto vulnerable en Lima, Perú.

\section{Metodología}

\subsection{Enfoque}

El estudio se basó en un enfoque cuantitativo, de alcance explicativo, tipo de diseño preexperimental con mediciones repetidas: pretest y postest (Tamayo y Tamayo, 2015), con el fin de medir los efectos de intervención programa de Educación Sensible en la convivencia escolar.

\subsection{Muestra}

La muestra y muestreo fueron no probabilísticos, ya que fueron los métodos más adecuados para el tipo de investigación aplicado en el estudio. La muestra se conformó por 40 estudiantes del cuarto grado de primaria de Educación Básica Regular del Perú ( $\mathrm{M}_{\text {(edad) }}=$ 10.1 años; D. E.= 1.84); a su vez, la distribución por sexo fue casi equitativa (masculino = $57.4 \%$, femenino = $42.6 \%$ ). De igual modo, para esta selección de sujetos, se optó por la organización de un muestreo no probabilístico de sujetos afines al estudio o por conveniencia para verificar que los efectos iniciales reportarán evidencias útiles para experimentos puros dignos de replicarse en el futuro con muestras similares. Las personas participantes eran estudiantes de instituciones educativas de gestión pública del distrito de Comas, originarios de la ciudad de Lima. Todos participaron bajo el procedimiento de consentimiento informado gestionado a través de la dirección de las escuelas y de los padres de familia. Cabe señalar que el estudio se organizó a inicios del año 2018 y se ejecutó hasta fines de noviembre del mismo año.

\subsubsection{Criterios de selección (inclusión)}

Debido a que en la Educación Básica Regular (EBR) del Perú se atiende a estudiantes con diversas necesidades educativas, se tuvo en cuenta la normativa EBR sobre la inclusión de sujetos con necesidades especiales, la cual establece como criterio obligatorio albergar a un máximo de dos estudiantes por aula con dichas necesidades. Este hecho tiene la 
finalidad de no acrecentar factores psicológicos sobre su tratamiento en el aula. Por ello, se decidió desarrollar el programa de acciones de Educación Sensible con la totalidad del alumnado (con y sin deficiencias). Sin embargo, para el registro de datos, fue importante obtener la ayuda del personal auxiliar en educación personalizada de cada aula para la atención de una cantidad de estudiantes caracterizados con:

- Trastorno con espectro autista leve (1 sujeto).

- Trastorno por déficit de atención e hiperactividad (1 sujeto).

Cabe señalar que esta investigación se desarrolló en el Sector 1 del contexto general de Comas, distrito de Lima, una vez comenzado el año escolar. La sociedad de este sector está caracterizada por distintos factores. Uno de ellos, es el económico, ya que los ingresos de sus padres y madres se encontraban por debajo de la media de consumo habitual familiar, lo cual desestabiliza en cierta medida las capacidades estudiantiles en el buen desarrollo escolar. Sin embargo, cada escuela participa en el programa nacional de alimentación de Perú otorgado por el Ministerio de Educación, lo cual permitió que la alimentación diaria fuera asegurada sin falta durante el desarrollo de la investigación. Otro factor de vulnerabilidad en el distrito se centra en la violencia social entre sus habitantes; una de ellas relacionadas con matanzas o resquebrajamiento social. Estas circunstancias podrían haber afectado al grupo estudiantil en su desarrollo socioafectivo; no obstante, se tomó como medida de control el trabajo con los padres de familia, ya que, al ser informados con anticipación, estos tomaron previsiones para asentar el clima escolar en los hogares y, con apoyo del Comité de Familia por Escuela se promovió la convivencia intrafamiliar para el seguimiento y realización del programa ES con total normalidad, tratando de disminuir los factores mencionados.

\subsection{Técnicas de recolección de datos y procedimiento}

Escala de medición de la convivencia democrática (ESMCD) (ad hoc). Es un instrumento elaborado para el estudio, de construcción politómica, y categorización ordinal. Esta escala de aplicación colectiva se aplicó bajo técnica de encuesta en un tiempo de resolución de 20 y 30 minutos (Ver anexo 1). El instrumento está compuesto por 25 proposiciones que buscan la medición de la convivencia democrática. Las respuestas para cada pregunta fueron: "Muy de acuerdo" = 1 punto; "De acuerdo" = 2 puntos y "En desacuerdo" = 3 puntos. Dicha escala midió las dimensiones: (a) colaboración y tolerancia, 
(b) normas de convivencia y (c) resolución de conflictos. La escala se sometió a un proceso de validación de expertos, mediante la participación de cinco (5) jueces evaluadores en la categoría de contenido, de lo cual se obtuvo un promedio de aceptación del 95.2 \%, por lo que el instrumento se consideró válido. De igual modo, se desarrolló el plan de pilotaje de la versión final del instrumento con 35 estudiantes del distrito de Comas, los cuales emitieron respuestas positivas sobre la escala y, luego del análisis estadístico, se obtuvo que la confiabilidad del instrumento fue aceptable (Tabla 1).

Tabla 1

Índice de confiabilidad Alfa de Cronbach del instrumento ESMD

Alfa de Cronbach N. de elementos

.889

25

Fuente: Elaboración propia con los datos recolectados, 2018

En el procedimiento de investigación, en un inicio, se gestionaron los permisos necesarios con la dirección de las escuelas integradas al estudio con dos meses de anticipación. Luego, para la verificación del instrumento, se plantearon dos modelos, con el fin de hacer comparables los veredictos del juicio de los cinco expertos que asumieron la evaluación de contenido. Por consiguiente, se decidió eliminar algunos ítems contenidos en "otros" y así otorgar mayor posibilidad de respuestas claras hacia cada pregunta (Tabla 2). Finalmente, el instrumento se aplicó a los 35 estudiantes destinados a pasar la encuesta en sus respectivas aulas. Los resultados finales permitieron que el instrumento se perfeccionara de tal modo que los ítems fueran más amigables y así mediar en la medición de la convivencia planteando proposiciones evaluativas.

Tabla 2

Extracto de ítems del instrumento de pilotaje aplicado en la evaluación previa a la investigación (dimensión colaboración y tolerancia)

\begin{tabular}{lc}
\hline \multicolumn{1}{c}{ Ítems } & $\begin{array}{c}\text { Alfa de } \\
\text { Cronbach } \\
\text { (índices } \\
\text { previos) }\end{array}$ \\
\hline 1. Compartes tus útiles escolares. & 0,605 \\
2. Compartes los juegos de mesa. & 0,403 \\
3. Utilizas los espacios escolares en la hora de recreo con respeto hacia los & 0,706 \\
demás. & \\
4. Actúas de forma colaborativa. & 0,890 \\
5. Cumples con acuerdos del aula. & 0,561 \\
\hline
\end{tabular}

Fuente: Elaboración propia con los datos recolectados, 2018 
Los resultados obtenidos inicialmente fueron importantes para la corrección y reformulación de ítems; por ejemplo, hacer adecuaciones gramaticales al tiempo presente (Tabla 2): "comparto útiles escolares", "comparto juegos de mesa", "utilizo..." con el fin de lograr la asimilación de ítems por parte de cada integrante con mayor confianza en la evaluación de la democracia. En cuanto al programa de Educación Sensible, este se estructuró con 40 actividades basadas en el enfoque de Herbart (en Touriñan, 2013), plasmadas en cuatro fases pedagógicas básicas para el desarrollo de la convivencia democrática mediante el programa de experimentación (Figura 1).

Figura 1. Explicativo del programa ES y sus productos prácticos en el aula para el desarrollo de la convivencia democrática

A

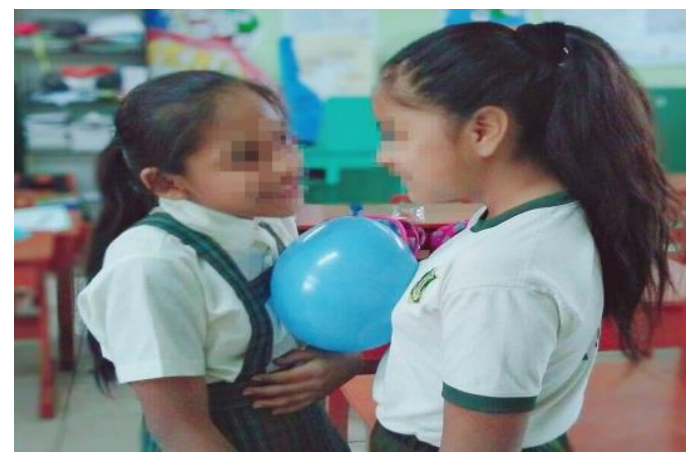

Fase pedagógica: conciencia sensible

B

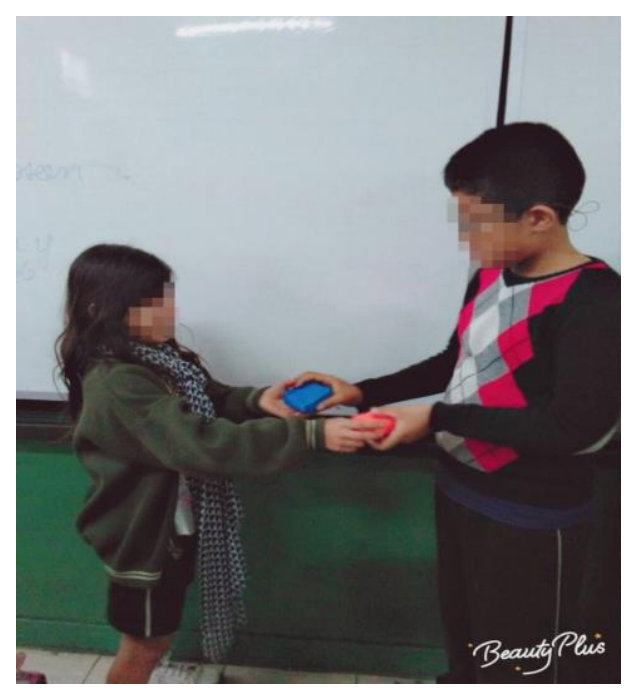

Fase pedagógica: autonomía emocional
C

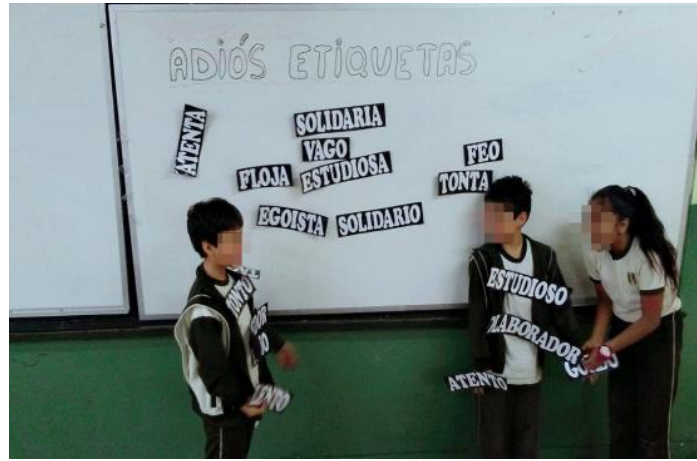

Fase pedagógica: autoevaluación emocional

D

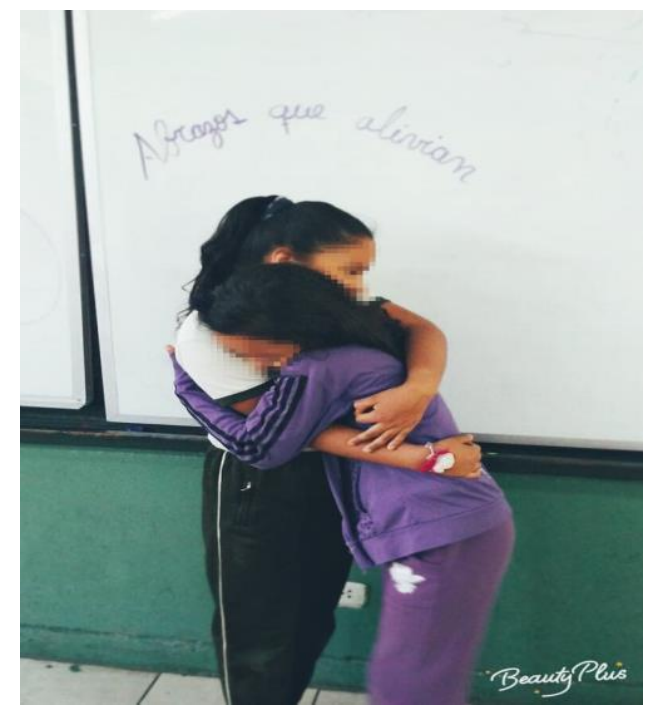

Fase pedagógica: sensibilidad aplicada

Fuente: Recursos de la investigación, 2018.

Nota: Actividades derivadas de las cuatro fases pedagógicas del programa ES: A = "no lo revientes"; B = "adiós etiquetas"; C = "hora de compartir"; $\mathrm{D}$ = "abrazos que alivian". 
Las actividades se desarrollaban tratando de respetar el orden de las fases

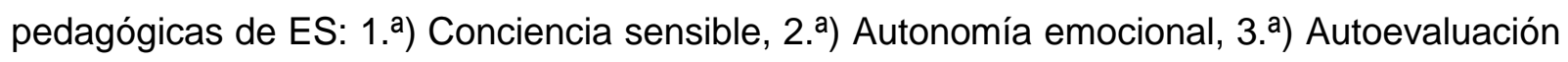
emocional y $4 .^{\underline{a}}$ ) Sensibilidad aplicada (Figura 1). La primera, exigió el desarrollo del sentido de proximidad mediante actividades retadoras, las cuales pusieran en práctica el conocimiento del espacio físico y espiritual entre en el grupo de participantes. Con la segunda, se consiguió que actuaran de forma individual, respecto de situaciones simuladas de rechazo para relacionarse mediante métodos de comunicación visoespacial y verbal. Como tercera fase, se estimuló la evaluación de los propios estados emocionales de cada integrante, analizándolos por comparación de experiencias desagradables. Por último, la sensibilidad aplicada fue la fase en que los sujetos comenzaron a comprender las emociones de otros y simular acciones gratas o eventos que generaran sentimientos agradables compartidos, lo cual permitió que volvieran a evaluar el sentido de proximidad mediante actividades físico-espirituales.

Las actividades descritas se homologaron al proceso curricular de cada docente a cargo, ya que cada uno desarrollaba sesiones de aprendizaje de acuerdo a enfoques de enseñanza similares, pero fue necesario aunar el enfoque de Educación Sensible mediante el desarrollo del programa bajo un solo objetivo para el desarrollo de la competencia democrática: "establecer la convivencia democrática desde una pedagogía basada en el autoconocimiento de la sensibilidad, tolerancia y análisis de la realidad para actuar con conciencia social".

Figura 2. Productos del programa ES mediante el desarrollo de sus fases pedagógicas para el logro de la convivencia

E

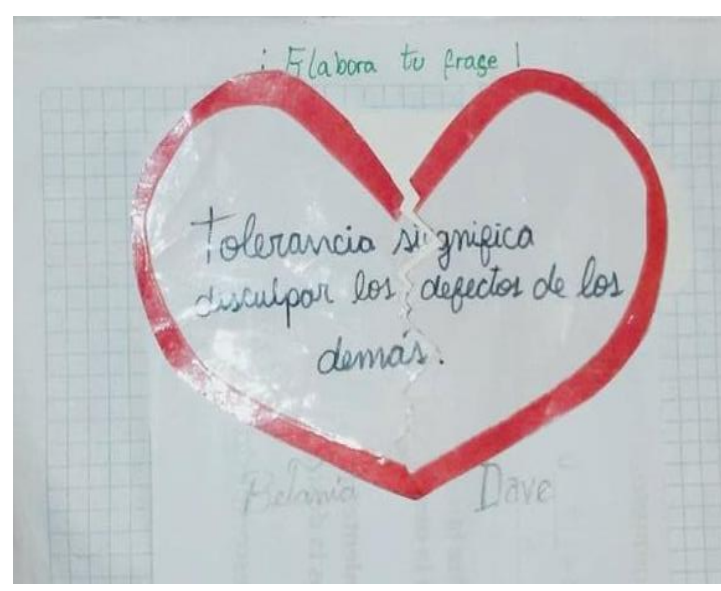

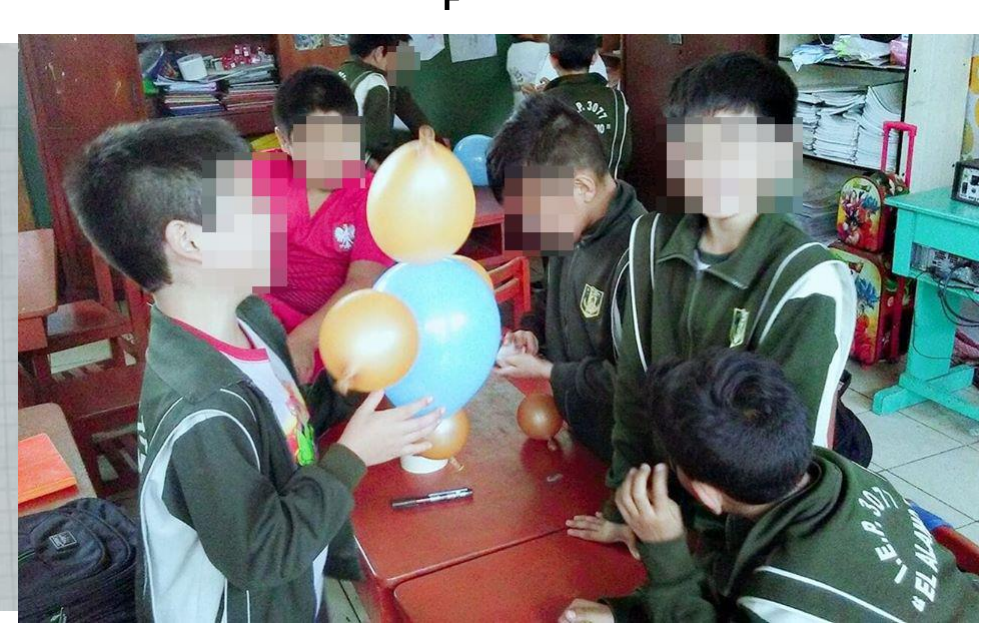

Fuente: Recursos de la investigación, 2018

Nota: Actividades: $\mathrm{E}=$ "mediamos juntos"; $\mathrm{F}$ = "hombre globo".

Los contenidos de este artículo están bajo una licencia Creative Commons 
Para el desarrollo de este programa, se implementó un aproximado de 160 tipos de materiales con el fin de desarrollar otros productos, como insignias de desarrollo de cada actividad (globos, cajas, cuerda, hojas, entre otros). Esto permitió la elaboración de otros productos propios de la interacción sensible en el aula (Figura 2), y las actividades de concientización de la democracia. Finalmente, el programa se desarrolló durante cuatro (4) meses, lo cual permitió el desarrollo adecuado de cada actividad para concretar los efectos de cada fase pedagógica en la convivencia democrática escolar.

\subsection{Procesamiento de análisis}

Para el procesamiento de datos, previamente se realizó el análisis de normalidad de los datos extraídos de la muestra de estudio sobre la convivencia democrática (CD). Para este propósito, se utilizó una prueba previa de análisis de datos (Kolmogorov-Smirnov) y se obtuvieron índices que señalaban una distribución no cercana a dicha normalidad en el cálculo de la variable (CD pre $(\mathrm{KS}=0.162) ; p<.05$; CD pos $(\mathrm{KS}=0.088) ; p>.05)$. Este contraste se realizó mediante el Test o Prueba de Rangos de Wilcoxon, ya que los datos no provenían de una distribución normal. La hipótesis nula para cada contraste se planteó desde la igualdad de medianas entre las mediciones realizadas antes y después del programa $E S\left(M d n_{\text {(pretest) }}=\right.$ $\left.M d n_{(\text {postest) }}\right)$ Finalmente, se decidió plantear un rango de decisión de $5 \%$ como significancia estadística o límite de error permitido $(p=0,005)$.

\section{Resultados}

\subsection{Educación sensible y convivencia democrática}

La hipótesis general a comprobar se generó sobre igualdad de medianas entre las mediciones pretest y postest $\left(M d_{1}=M d_{2}\right): \mathrm{h}_{(\mathrm{o})}=$ los efectos del programa Educación Sensible no generan diferencias significativas en las medianas provenientes de las puntuaciones de la convivencia democrática en escolares del cuarto grado de primaria. Por lo tanto, la hipótesis alterna fue: $\mathrm{h}_{(\mathrm{i})}=$ los efectos del programa Educación Sensible generan diferencias significativas en las medianas provenientes de las puntuaciones de la convivencia democrática en escolares del cuarto grado de primaria. También se siguió similar estructura para el contraste de otras hipótesis basadas en las dimensiones colaboración y tolerancia, normas de convivencia y resolución de conflictos.

Los resultados esperados en el cambio de la convivencia democrática bajo la intervención de la educación sensible fueron efectivos en el grupo estudiado. En un primer 
contraste, se encontraron puntuaciones positivas respecto de la efectividad del programa ES sobre la convivencia democrática. Hubo mejoras en los 40 sujetos del estudio, de los cuales se obtuvo 20.50 de rango promedio y 820.00 en la suma de rangos $\left(M d_{1} \neq M d_{2} ; Z=-5,513 ; p\right.$ $<0,05$ ) (Tabla 3). Estas diferencias permitieron aceptar la hipótesis de diferencia de medianas (h.alterna): $\mathrm{h}_{(\mathrm{i})}=$ los efectos del programa Educación Sensible generan diferencias significativas en las medianas provenientes de las puntuaciones de la convivencia democrática en escolares del cuarto grado de primaria. Algunos estudios, señalan que los mecanismos para sensibilizar, producir estímulos para la autonomía y la autoevaluación de las emociones producen cambios para la convivencia en contextos de problemática social y política (Grau et al., 2016; Grau y García-Raga, 2016), y son desencadenantes de interrelaciones saludables. Esto es un aspecto motivacional importante para el desarrollo humano, así como para el crecimiento valorativo estudiantil que desarrollan al egresar de la escuela.

El programa ES ha surtido efectos iniciales en el cambio de la convivencia desde un estilo democrático. Como se pudo ver en estos resultados, los procesos culturales cohesivos del desarrollo de las cuatro fases pedagógico-formativas del programa han permitido mediatizar en el sentido práctico de convivencia escolar, es decir, no solo se han centrado en saber, sino también en ejecutarla con criterio de convivencia (Berlowitz et al., 2017; DeAngelis, 2017).

Tabla 3

Diferencias en distribución y significancias entre las mediciones pretest y postest en la variable convivencia democrática y sus dimensiones

\begin{tabular}{|c|c|c|c|c|c|c|c|}
\hline & & \multicolumn{6}{|c|}{ Dimensiones } \\
\hline \multicolumn{2}{|c|}{$\begin{array}{l}\text { Convivencia } \\
\text { democrática }\end{array}$} & \multicolumn{2}{|c|}{$\begin{array}{c}\text { Colaboración y } \\
\text { tolerancia }\end{array}$} & \multicolumn{2}{|c|}{$\begin{array}{l}\text { Normas de } \\
\text { convivencia }\end{array}$} & \multicolumn{2}{|c|}{$\begin{array}{c}\text { Resolución de } \\
\text { conflictos }\end{array}$} \\
\hline Z & $-5,513$ & Z & $-5,162$ & Z & $-5,437$ & Z & $-5,385$ \\
\hline Sig. & 0.0001 & Sig. & 0.0001 & Sig. & 0.0001 & Sig. & 0.0001 \\
\hline
\end{tabular}

Fuente: Elaboración propia con los datos recolectados

Nota: $Z$ = diferencia de distribución; sig.= significancia bilateral

En cuanto al contraste en la dimensión colaboración y tolerancia (Tabla 3 ), se evidenciaron 35 mejoras en el grupo: cuatro sujetos con estabilidad (ni cambio ni rechazo) y una puntuación negativa (rango promedio = 18.87; suma de rangos $=660.50) ;\left(M d_{1} \neq M d_{2} ; Z\right.$ $=-5,162 ; p<0,05)$. De modo similar, también se comprobó la diferencia de medianas: $\mathrm{h}_{(\mathrm{i})}=$ los efectos del programa Educación Sensible generan diferencias significativas en las 
medianas provenientes de las puntuaciones de la dimensión colaboración y tolerancia como parte de la convivencia democrática en escolares del cuarto grado de primaria. En relación a la dimensión normas de convivencia (Tabla 3), se puede afirmar que este cambio también ha sido significativo, con 38 mejoras individuales en la muestra (rango promedio = 20.50; suma de rangos $=779.00)$. Sin embargo, también se halló una diferencia negativa y un empate en el grupo de individuos participantes. La diferencia de medianas también se ha comprobado por los resultados obtenidos en cuanto a la significancia $\left(M d_{1} \neq M d_{2} ; Z=-5,437 ; p<0,05\right)$, por lo que se aceptó la hipótesis alterna del estudio: $\mathrm{h}_{(\mathrm{i})}=$ los efectos del programa Educación Sensible generan diferencias significativas en las medianas provenientes de las puntuaciones de la dimensión normas de convivencia como parte de la convivencia democrática en escolares del cuarto grado de primaria. Sin embargo, hay que notar que la falta de cambio en algunos sujetos no determinados con mejora en la progresión del grupo atendido se debe al desarrollo complejo del pensamiento democrático en cada escolar, más aún si se intenta desarrollar la convivencia democrática desde un perfil proactivo estudiantil (DeAngelis, 2017). En cierta medida, ha sido difícil para este tipo de estudiantes independizarse desde la perspectiva emocional. En este caso, la convivencia democrática influenciada por los efectos de ES es más prominente si cada escolar llega a razonar de forma autónoma, así como se buscó desde la fase de autonomía emocional del programa. Algunos sujetos presentaron dificultad para el logro de habilidades de cuestionamiento y reflexión. Ello ha sido crucial para verificar si el cambio desde un aspecto reflexivo también se ejerció mediante la Educación Sensible.

Por último, la resolución de conflictos (Tabla 3) ha presentado cambios a nivel estadístico como también a nivel descriptivo (rango promedio $=19.50$; suma de rangos = 741.00) y esto se ha obtenido en 38 individuos. Aunque no se evidenciaron cambios significativos en dos sujetos de la muestra, si fue significativo para el grupo en general $\left(M d_{1} \neq\right.$ $\left.M d_{2} ; Z=-5,385 ; p<0,05\right)$. Por lo tanto, se logró aceptar la hipótesis alterna en este contraste: $\mathrm{h}_{(\mathrm{i})}=$ los efectos del programa Educación Sensible generan diferencias significativas en las medianas provenientes de las puntuaciones de la dimensión resolución de conflictos como parte de la convivencia democrática en escolares del cuarto grado de primaria. Como se puede verificar, los efectos de la Educación Sensible se han relegado en estos dos sujetos. En este caso, las distintas formas de transponer sus actividades cotidianas a la escuela han permitido a muchos sujetos lograr el cambio sobre el manejo de conflictos como en otras investigaciones (DeAngelis, 2017; Samuelsson, 2016). Sin 
embargo, algunos no realizaban una práctica vivencial sobre el manejo en sus aulas, lo cual ha retrasado el desarrollo de la convivencia democrática y, por ende, el logro de indicadores que suplieran esta dimensión de manejo de conflictos. Esto es mucho más difícil de desarrollar si se toma en cuenta que la convivencia de estilo democrático se reproduce en grupos con cierta apertura hacia la autoevaluación y sensibilidad.

Desde una mirada contextual, se puede asumir que antes de iniciar el programa ES, las características de los escolares limeños se representaron por ser autoritarios, conflictivos y con cierta desorganización. Otros aspectos que, en paralelo, también influyeron en el desarrollo estudiantil, algunos de cuyos efectos se visualizaron en la aceptación del enfoque curricular, la organización educativa y la propuesta de convivencia. Cabe señalar que, ante estos efectos, aún es inaceptable para los directivos asumir que los problemas coyunturales de la sociedad son transversales, se originan en la población y se transfieren al alumnado desde su proximidad a la sociedad. En principio, desde los proyectos educativos institucionales se debería ejercer una educación integrada sobre temas sociales transversales. Hoy estos temas parecen ya no vincularse a la programación curricular ni a la pedagogía social. Muchos escolares en Lima suelen elegir autoridades del aula como brigadieres y policías escolares desde distintos procesos democráticos, por ejemplo, votaciones y foros. Sin embargo, quizá estas modalidades de elección hayan sido solo teóricas o, mejor dicho, verticales, lo cual impide que el estudiantado genere algún pensamiento democrático en su vida escolar. En este caso, el profesorado aún no muestra rasgos evolutivos sobre la didáctica del área Persona y Sociedad, por lo que estos microprocesos quedan relegados a ejecutarse como una actividad sin significado para el estudiantado.

Respecto a la organización educativa y la propuesta de convivencia en el centro escolar, se encuentran dos obstáculos permanentes para el profesorado y el grupo estudiantil: los acuerdos y la coacción. En el primero, es complejo para el grupo escolar llegar a cumplir acuerdos en contextos de convivencia, generalmente, agresivos y conflictivos. En cierta medida, la dificultad aparece porque cada estudiante tiene limitaciones para manejar ciertas habilidades emocionales y de autorregulación. Otra suele darse en las interrelaciones básicas, en las cuales se transmiten patrones de conducta agresiva repetitivos en diferentes grupos estudiantiles. En cuanto a la coacción, el sistema de supervisión docente infringe en la convivencia pacífica en el profesorado, lo cual ha originado la aparición de otras variables extrañas (miedo a la evaluación o a la continuidad docente). Al 
parecer este tipo de conductas se transmiten a cada escolar, en ambos casos, las traducen como comportamientos aversivos hacia el proceso educativo (evaluaciones continuas, aprendizajes complejos, tareas académicas).

Por lo tanto, existe un sistema de coacción que influye en los procesos de interrelación entre el profesorado y el alumnado o entre el propio estudiantado. En este caso, las actividades pedagógicas del programa ES trataron de acercar más a las estudiantes y los estudiantes con métodos de autoevaluación emocional y acercamiento físico espiritual, como capacidades previas para generar un mejor clima en el entorno de convivencia democrática del aula.

\subsection{Indicadores y limitaciones}

El análisis particular de la convivencia democrática se ha observado con evidente contundencia luego de la realización del programa ES (Tabla 4). De acuerdo a la Tabla 4, se revelan diferencias entre las puntuaciones promedio de las mediciones pretest y postest en la dimensión colaboración y tolerancia (4.45), como también en la dimensión normas de convivencia (9.35). De igual modo, surgieron para la dimensión resolución de conflictos (6.25).

Tabla 4

Medidas pretest y postest de las puntuaciones en dimensiones colaboración y tolerancia, normas de convivencia y resolución de conflictos en estudiantes de cuarto grado de primaria, Comas, Lima, Perú, 2018

\begin{tabular}{ccccccc}
\hline & \multicolumn{3}{c}{ Pretest } & & \multicolumn{3}{c}{ Postest } \\
\cline { 2 - 7 } Medidas & $\begin{array}{c}\text { Colaboración y } \\
\text { tolerancia }\end{array}$ & $\begin{array}{c}\text { Normas de } \\
\text { convivencia }\end{array}$ & $\begin{array}{c}\text { Resolución de } \\
\text { conflictos }\end{array}$ & $\begin{array}{c}\text { Colaboración y } \\
\text { tolerancia }\end{array}$ & $\begin{array}{c}\text { Normas de } \\
\text { convivencia }\end{array}$ & $\begin{array}{c}\text { Resolución } \\
\text { de } \\
\text { conflictos }\end{array}$ \\
\hline Media & 9.65 & 12.20 & 9.05 & 14.10 & 21.55 & 15.30 \\
D.E.* & 1.79 & 2.15 & 1.96 & 2.93 & 4.33 & 2.49 \\
Varianza & 3.21 & 4.63 & 3.84 & 8.61 & 18.77 & 6.22 \\
\hline
\end{tabular}

Fuente: Elaboración propia con los datos recolectados, 2018. Nota: *desviación estándar.

A pesar de ello, es importante dar cuenta de algunos indicadores sin mejora significativa y con mayor dificultad para desarrollarse, como "actúo de forma colaborativa en el trabajo en equipo" (Figura 3), que aunque presenta porcentajes mayores a $50 \%$ en el análisis de las mediciones pretest y postest, ha sido una de las características más difíciles de desarrollar en el grupo analizado. Esto refleja que en el contexto vulnerable desarrollado también se gestan ciertos patrones conductuales asociados a valores negativos, que algunos de los sujetos se dedicaban a desarrollar trabajos muy personalistas o establecer el cómo rendir o rendir más en lugar de considerar el progreso de otros. 
Figura 3. Porcentaje del estudiantado por indicadores de convivencia democrática, según nivel de acuerdo, comparación del programa ES (pretest-postest), Comas, Lima, Perú, 2018

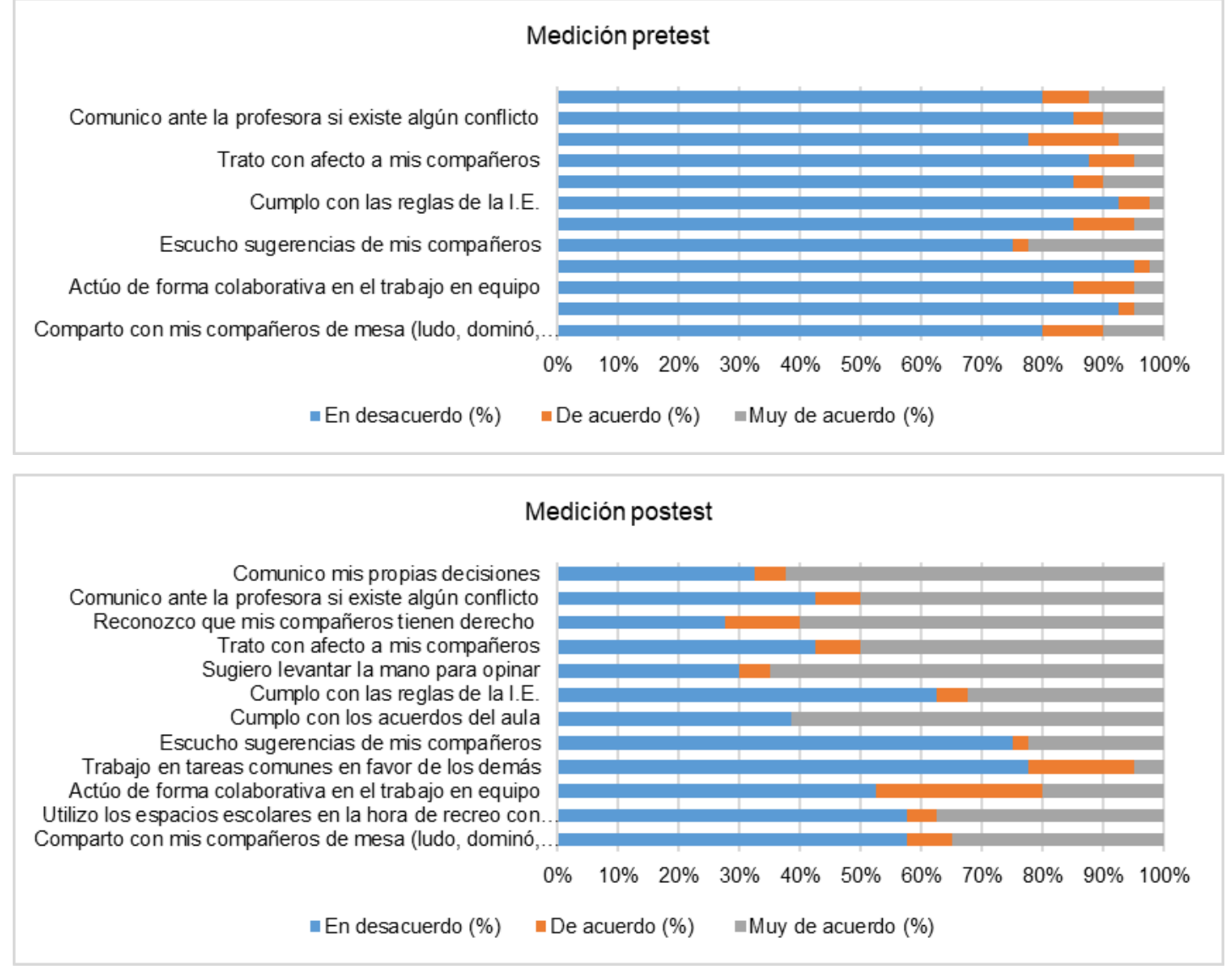

Fuente: Elaboración propia con los datos recolectados, 2018.

El indicador "reconozco que mis compañeros tienen derecho" describe cómo el estudiantado reconoce el derecho de otros. No obstante, para cada participante fue ciertamente un indicador complejo de concientizar, aunque comprendieron que es importante para el logro de la justicia social. Ante este hecho se percibió que la mínima cantidad de sujetos al finalizar el programa ES presentó resistencia a reconocer el derecho de otros, sobretodo en situaciones emocionalmente más interactivas, que a su vez implicaban el uso de proximidad física. Los individuos de este experimento deben madurar el sentido de reconocimiento del derecho y sus conceptos: qué es derecho, cómo se compone como parte de la humanidad.

Otro aspecto importante que todavía deberá exigirse es el criterio de compartir "comparto con mis de compañeros de mesa...". Este se ha reflejado como una forma valorativa de la convivencia en el modo más concreto posible de acuerdo a las acciones que 
el grupo estudiantil presentó. En cierta medida, desarrollar la autonomía emocional y autoevaluarse son aspectos que se deben a la adecuación pedagógica de las normas ante situaciones estudiantiles específicas que las personas participantes desarrollaron (Alaníz Castro et al., 2016; Berlowitz et al., 2017; Eriksen, 2018; Gass et al., 2016; Grau et al., 2016). No obstante, este valor axiológico se adhiere al sistema de convivencia de cada escolar en cuanto exista un peso determinado de dicha autonomía y autoevaluación en los sujetos participantes. En cierta medida, este valor puede desarrollarse a la par de la convivencia democrática, pero su implicancia no se determina por obligatoriedad, sino por medio del sistema actitudinal escolar, el cual es el determinante para convivir y compartir la democracia con otros.

Como limitación, es importante señalar que, aunque se hayan previsto las condiciones económicas y psicosociales para cada integrante de la muestra, la predisposición del colectivo docente fue negativa durante el desarrollo de la investigación. En cierta medida, porque asumían que el desarrollo de la convivencia democrática en el aula no se percibiría de modo positivo por las autoridades de las instituciones educativas (directores 0 coordinadores de grado). Es inevitable desestimar que el profesorado presentó pocas actitudes hacia la democracia; sin embargo, estas se transmitieron al grupo estudiantil, el cual, por ciertas razones, sí estaban predispuestos hacia la democracia. Cabe señalar que eran sujetos acondicionados a las formas de convivencia del equipo docente y su modalidad autoritaria era evidente en muchos casos. El programa tuvo dificultades en la transformación de la convivencia escolar. Esta fue una de las razones por las que el desarrollo en cada escolar de la democracia en convivencia fuera tensa y duradera, sobre todo para la construcción de su consciencia en estos hábitos de vida.

\section{Conclusiones}

Respecto de la variable convivencia democrática, entre las mediciones comparadas (pretest y postest), los cambios fueron significativos en el grupo de experimentación (rango promedio $=820.00 ; p<.05)$. Los sujetos de estudio desarrollaron patrones de autoconciencia y autoevaluación emocional mediante la Educación Sensible en el programa educativo.

Las actividades de sensibilidad afectiva, conductual y actitudinal predispusieron a los sujetos de experimentación en el desarrollo de la dimensión la colaboración y tolerancia (rango promedio $=660.50 ; p<.05$ ). Los sujetos demostraron mejor capacidad para compartir 
el trabajo en equipo al tiempo que aprendieron a escuchar sugerencias con mayor aplomo en el aula. En este caso, han sido mucho más efectivas las técnicas de autoevaluación emocional y conciencia sensible, puesto que los sujetos necesitaban conocerse entre sí, comunicarse y, sobretodo, comprender el estado emocional con que lo hacían a diario. Estas técnicas han favorecido el cuestionamiento de su proximidad física y espiritual.

En cuanto a la dimensión normas de convivencia, se establecieron mejoras luego de la aplicación del programa de Educación Sensible (rango promedio $=779.00 ; p<.05$ ). Los y las participantes de la muestra presentaron mejor reflexividad ante las normas, como también compartir alguna reflexión a otros compañeros y hacerlo con mayor constancia. De acuerdo a estos resultados, la sensibilidad aplicada les ha permitido comprobar de modo práctico su capacidad de proximidad, estudiar el pensamiento de otros para compartirlo o saber cuándo cooperar en la distribución del poder, en este caso, poder democrático.

En cuanto a la dimensión resolución de conflictos, luego de la aplicación del programa $E S$, se encontraron diferencias significativas (rango promedio $=741.00 ; p<.05$ ). Los individuos participantes aprendieron proponer soluciones, abordar conflictos de forma pacífica y tomar decisiones más alturadas. Esto provino del sentido sensible y autoevaluación emocional que desarrolló el programa en fases pedagógicas formativas de la convivencia democrática.

Respecto del programa ES, es necesario concluir que el conocimiento en cada participante, la naturaleza de su convivencia democrática y las formas de interacción entre estudiantes y docentes permitieron plantear actividades pedagógicas sensibles basadas en los postulados de Herbart y Lowe, sustentables para ajustar actitudes y comportamientos autoritarios a modelos de comportamiento democrático. También se concluye que el colectivo docente necesita generar un modelo o enfoque pedagógico basado en la crítica social. Este propósito permitiría desarrollar pensamientos críticos ajustados a una realidad sin manipulaciones o desvíos políticos, como ocurre en la sociedad peruana actual.

Por último, es necesario señalar que la programación curricular necesita realizarse desde diagnósticos sustentados en coyunturas sociales. Cada docente con habilidades para la programación pedagógica, necesitan utilizar técnicas como la matriz FODA (fortalezas, amenazas, debilidades, amenazadas) aplicada, sobre todo, a contextos como el de esta investigación. El objetivo es reformular necesidades formativas sociales y temas transversales, que permitan que la programación a corto plazo (sesiones de aprendizaje, 
módulos, proyectos) se aborde en busca del fortalecimiento del pensamiento democrático y, por ende, de su convivencia escolar.

\section{Reflexiones finales}

Como reflexión propia, los autores expresan su sentir desde el discurso pedagógico, no sin antes haber comprendido tres dimensiones humanas para la generación de democracia en el grupo estudiantil: comprensión de la otredad, espacio-proximidad y toma de conciencia. Esto nos permitió analizar y valorar dichos aspectos con el fin de comprender, en primer lugar, la existencia de otros en nuestro entorno, a quienes expresamos nuestros pensamientos y también escuchamos. Desde este principio, se puede colegir que, como competencia previa para el desarrollo de la convivencia democrática, es importante conocer a cada escolar y al profesorado en común: destrezas, emociones y actitudes con el fin de establecer normas compartidas en un entorno y clima democrático.

El espacio-proximidad nos ayudó a comprender que el colectivo estudiantil necesita de entornos socialmente abiertos, flexibles y adaptables a situaciones, lugares y tiempos de un contexto determinado. Aunque en este caso, el contexto de vulnerabilidad ha sido crucial en que los efectos del programa fueran sostenibles, es importante señalar el cambio encontrado en cada persona participante de este estudio, así como en el trabajo en equipo. Los niños y las niñas necesitan conocer el espacio físico y espiritual con el fin de conocer el derecho de otros, los limites como principal medio para convivir, pues la convivencia no se produce sin ejercerla, es más, no sin conocerla previamente a profundidad en el grupo estudiantil y en la práctica docente. Por último, la toma de conciencia permitirá que cada integrante de la escuela pueda aprender desde un enfoque de pensamiento crítico y sociocrítico, y desarrollar actitudes y comportamientos prosociales ante la comunidad, pues la convivencia será el nexo entre sociedades sostenibles y las organizaciones que las dirigen con el fin de mejorar los entornos de aprendizaje escolar. 


\section{Referencias}

Aguilar, Guido., Peter de Bran, Monika. y Aragón de Herrante, Luisa (2014). Desarrollo de habilidades sociales en niños y adolescentes. México: Trillas.

Alaníz Castro, Dorling María., Morán López, Judith Azalia. y Blandón Blandón, Judith del Carmen. (2016). Estrategias que favorecen la participación en niños/as de III nivel de la Escuela Emmanuel Mongalo y Rubio del municipio de Yalí, durante el año 2015 (Tesis de licenciatura). Universidad Nacional Autónoma de Nicaragua, Nicaragua. Recuperado de http://repositorio.unan.edu.ni/1767/

Aslan, Serkan. (2018). Relationship between the Tendency to Tolerance and Helpfulness Attitude in 4th Grade Students. International Journal of Progressive Education, 14(2), 29-36. doi: https://doi.org/10.29329/ijpe.2018.139.3

Asociación Internacional para la Evaluación de Logros Educativos. (2016). Estudio Internacional de Cívica y Ciudadanía. Recuperado de http://umc.minedu.gob.pe/iccs/

Berlowitz, Marvin J.; Frye, Rinda. y Jette, Kelli M. (2017). Bullying and Zero-Tolerance Policies: The School to Prison Pipeline. Multicultural Learning and Teaching, 12(1), 725. doi: http://dx.doi.org/10.1515/mlt-2014-0004

Bickmore, Kathy., Salehin Kaderi, Ahmed. y Guerra-Sua, Ángela. (2017). Creating capacities for peacebuilding citizenship: history and social studies curricula in Bangladesh, Canada, Colombia, and México. Journal of Peace Education, 14(3), 282-309. doi: https://doi.org/10.1080/17400201.2017.1365698

Bowie, Robert. (2017). Is tolerance of faiths helpful in English school policy? Reification, complexity, and values Education. Oxford Review of Education, 43(5), 536-549. doi: http://dx.doi.org/10.1080/03054985.2017.1352350

Chang, Jina y Song, Jinwoong. (2016). A case study on the formation and sharing process of science classroom norms. International Journal of Science Education, 38(5), 747-766. doi: http://dx.doi.org/10.1080/09500693.2016.1163435

Colcord, Cean R., Mathur, Sarup P. y Zucker, Stanley H. (2016). Improving disciplinary practices in an urban school: Solving the problem of practice, Journal of Education and Training Studies, 4(10), 144-155. doi: https://doi.org/10.11114/jets.v4i10.1811

DeAngelis, Corey A. (2017). Do self-interested schooling selections improve society? A review of the evidence. Journal of School Choice, 11(4), 546-558. doi: http://dx.doi.org/10.1080/15582159.2017.1395615

Dewey, John. (1916). Democracy and education: an introduction to the philosophy of education. New York, United States: MacMillan.

Eriksen, Kristin Gregers. (2018). Bringing Democratic Theory into Didactical Practice. Concepts of Education for Democracy Among Norwegian Pre-service Teachers. Interchange, 49(3), 393-409. doi: http://dx.doi.org/10.1007/s10780-018-9332-7 
Gass, Michael., Gough, Sarah., Armas, Andres. y Dolcino, Cristina. (2016). Play for Peace as a Violence Prevention Model: Achieving Voluntad y Convivencia. Journal of Experiential Education, 39(4), 412-426. doi: https://doi.org/10.1177/1053825916674978

Grau, Roser. y García-Raga, Laura. (2017). Learning to live together: a challenge for schools located in contexts of social vulnerability. Journal of Peace Education, 14(2), 1-18. Doi: https://doi.org/10.1080/17400201.2017.1291417

Grau, Roser., García-Raga, Laura. y López-Martín, Ramón. (2016). Towards School Transformation. Evaluation of a Coexistence Program from the Voice of Students and Teachers. Journal of New Approaches in Educational Research, 5(2), 137-146. doi: https://doi.org/10.7821/naer.2016.7.177

Jakobus Jacobs, Petrus. (2015). School environment as moderator in the relationship between school engagement, coping and resilience among South African adolescents (Tesis de Maestría). University of the Free State, Sudáfrica. Recuperado de http://scholar.ufs.ac.za:8080/xmlui/bitstream/handle/11660/4624/JacobsPJ.?sequence= 1\&isAllowed $=y$

Lowe, Pam. (2015). Lessening sensitivity: student experiences of teaching and learning sensitive issues. Teaching in Higher Education, 20(1), 119-129. Doi: http://dx.doi.org/10.1080/13562517.2014.957272

Muñoz Quezada, María Teresa., Lucero Moncada, Boris Andrés., Cornejo Araya, Claudia Alejandra., Muñoz Molina, Pablo Andrés. y Araya Sarabia, Nelson Eduardo. (2014). Convivencia y clima escolar en una comunidad educativa inclusiva de la Provincia de Talca, Chile. Revista Electrónica de Investigación Educativa, 16(2), 17-23. Recuperado de: https://redie.uabc.mx/redie/article/view/497/923

Proyecto Educativo Metropolitano. (2014). Lima Ciudad Educadora, amiga de los niños y las niñas. Lima, Perú: Municipalidad Metropolitana de Lima.

Redford, Jeremy; Desrochers, Donna; Hoyer, Kathleen Mulvaney. (2017). The Years before School: Children's Nonparental Care Arrangements from 2001 to 2012. Stats in Brief. NCES 2017-096. National Center for Education Statistics. Recuperado de https://files.eric.ed.gov/fulltext/ED573320.pdf

Rendón, María Alexandra., Cuadros, Olga Elena., Hernández, Beatriz Elena., Monterrosa, Diana Cecilia., Holguín, Amparo., Cano, Lina María., Álvarez, Johanna Patricia. y Ortiz, Adriana María. (2016). Las competencias socioemocionales en el contexto escolar. Medellín, Colombia: Editorial Universidad de Antioquia.

Samuelsson, Martin (2016). Education for Deliberative Democracy A Typology of Classroom Discussions. Democracy \& Education, 24(1), 1-9. Recuperado de https://democracyeducationjournal.org/home/vol24/iss1/5/

Schunk, Dale H. (2012). Teorías del aprendizaje: una perspectiva educativa (6a. ed.). México: Pearson Educación. 
Tamayo y Tamayo, Mario. (2015). El proceso de investigación científica. Balderas, México: Limusa.

Touriñan, José Manuel. (2013). El significado de la función pedagógica y la necesidad de generar principios de acción. Revista española de pedagogía, 71(254), 29-47. Recuperado de https://revistadepedagogia.org//xxi/no-254/el-significado-de-la-funcionpedagogica-y-la-necesidad-de-generar-principios-de-accion/101400010286/ 


\section{Anexo 1}

\section{Escala de medición de la convivencia democrática (ESMCD)}

(Versión final)

\section{Datos personales:}

Género: $M-F$

Edad:

Grado:

Marca con un aspa $(\mathrm{x})$ para cada respuesta.

\begin{tabular}{|c|c|c|c|}
\hline \multirow[b]{2}{*}{ Ítems* } & \multicolumn{3}{|c|}{ Escala de valoración } \\
\hline & $\begin{array}{c}\text { En } \\
\text { desacuerdo }\end{array}$ & De acuerdo & $\begin{array}{l}\text { Muy de } \\
\text { acuerdo }\end{array}$ \\
\hline \multicolumn{4}{|l|}{ 1.Comparto mis útiles escolares } \\
\hline \multicolumn{4}{|l|}{ 2. Comparto los juegos de mesa. } \\
\hline \multicolumn{4}{|c|}{$\begin{array}{l}\text { 3.Utilizo los espacios escolares en la hora de recreo con respecto } \\
\text { hacia los demás }\end{array}$} \\
\hline \multicolumn{4}{|l|}{ 4.Actúo de forma colaborativa } \\
\hline \multicolumn{4}{|l|}{ 5.Cumplo con acuerdos del aula } \\
\hline \multicolumn{4}{|l|}{ 6.Comunico mis decisiones con libertad } \\
\hline \multicolumn{4}{|l|}{ 7.Los demás me permiten decir mis decisiones. } \\
\hline \multicolumn{4}{|c|}{ 8.Cuido a mis compañeros(as) de las decisiones que tomo y realizo } \\
\hline \multicolumn{4}{|c|}{ 9.Intervengo en conflictos de mis compañeros (as) para ayudar } \\
\hline \multicolumn{4}{|c|}{ 10.Evito maltratar a mis compañeros (as) } \\
\hline \multicolumn{4}{|l|}{ 11.Es importante convivir en armonía } \\
\hline \multicolumn{4}{|l|}{ 12.Propongo que conversemos adecuadamente } \\
\hline \multicolumn{4}{|l|}{ 13.Sugiero a otros levantar la mano para opinar } \\
\hline \multicolumn{4}{|c|}{ 14.Todos (as) debemos decidir sobre la tarea grupal por igual } \\
\hline \multicolumn{4}{|c|}{$\begin{array}{l}\text { 15.Comunico sobre algún conflicto (a algún profesor o alguna } \\
\text { profesora a cargo) }\end{array}$} \\
\hline \multicolumn{4}{|c|}{$\begin{array}{l}\text { 16.Comunico si no dejan hablar a mi compañero(a) (a algún } \\
\text { profesor o alguna profesora a cargo). }\end{array}$} \\
\hline \multicolumn{4}{|l|}{ 17.Escucho con atención las sugerencias de otros } \\
\hline \multicolumn{4}{|l|}{ 18.Propongo soluciones a algún conflicto de aula } \\
\hline \multicolumn{4}{|l|}{ 19.Escucho las soluciones de los(as) demás } \\
\hline \multicolumn{4}{|l|}{ 20.Realizo mi votación sin que me obliguen } \\
\hline \multicolumn{4}{|c|}{$\begin{array}{l}\text { 21.Converso si debo compartir un espacio en el recreo con otros } \\
\text { (as) }\end{array}$} \\
\hline \multicolumn{4}{|c|}{ 22.Pido permiso para usar un espacio para jugar } \\
\hline \multicolumn{4}{|l|}{ 23.Resuelvo mis conflictos sin agredir } \\
\hline \multicolumn{4}{|l|}{ 24.Opino sobre las reglas de mi escuela } \\
\hline 25.Realizo trabajos en grupo y todos(as) participan & & & \\
\hline
\end{tabular}


Revista indizada en

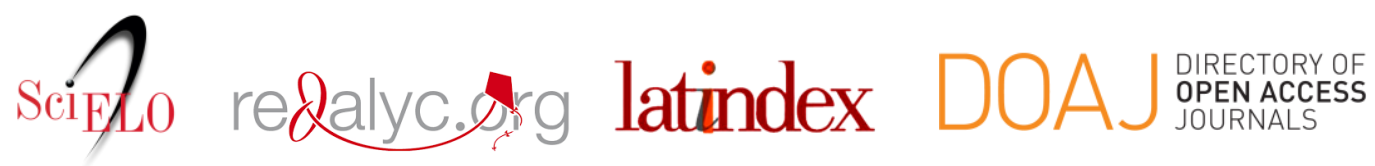

Distribuida en las bases de datos:

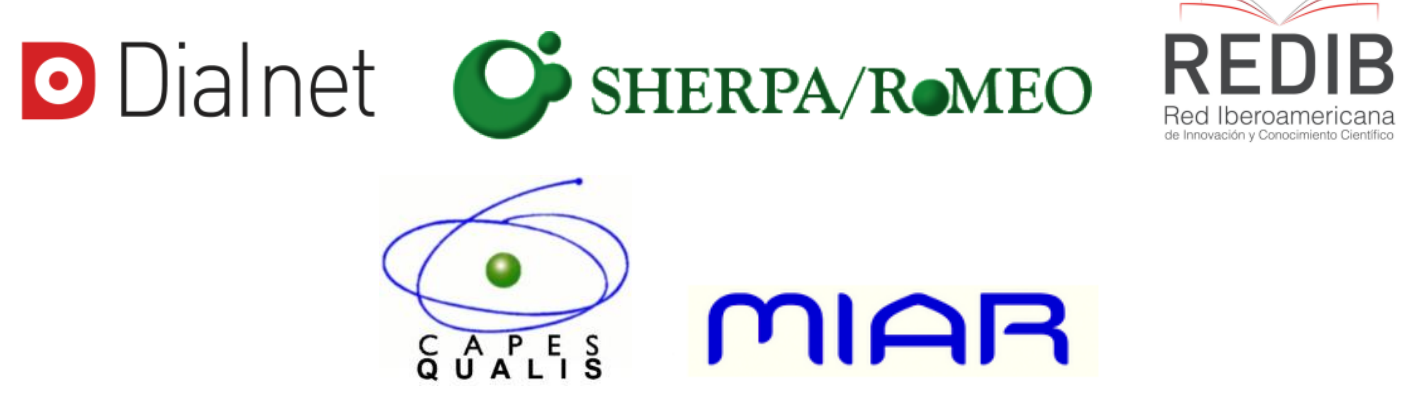

